\title{
Double Ring Firing Single Optical Fiber Deflector
}

\author{
A. A. Seymen, E. U. Şimşek, E. Özgür, and B. Ortaç®
}

\begin{abstract}
We report a novel fabrication method of double ring firing single optical fiber deflector. The fiber deflector is fabricated as a convergent cone having two different cone angles, by conventional mechanical polishing. The two-angled conical fiber deflector geometry is formed in a single fiber tip, deflecting incoming laser light into two distinct circular rings. Two cone angles provide two deflection angles due to total internal reflection and refraction of the incoming laser light. We achieved two deflected circular beams at $60^{\circ}$ and $85^{\circ}$ with the cone angles of $56^{\circ}$ and $72^{\circ}$ at one single fiber deflector. The laser power is evenly split between two conical areas. The fabrication process of the fiber deflector does not involve any splicing or arc discharge process; thus, it is convenient, effective, and high-throughput.
\end{abstract}

Index Terms-Laser ablation, light deflectors, optical fiber devices.

\section{INTRODUCTION}

O PTICAL fibers are widely used in surgical treatments for varicose vein surgery, lithotripsy, and photothermal therapy by use of laser light for ablation or irradiation of tissues [1]-[7]. Optical fibers could transmit the laser energy to desired tissue in the human body, since they are small in diameter, flexible, biocompatible, and have light-manipulation capabilities to various directions or forms with different deflector geometry. Three different optical fiber deflector designs have been used for the applications mostly for medical purposes: bare, side firing and radial. Bare fiber optic deflectors deliver the laser light in the same direction with optical fiber axis, where side-firing fiber optic deflectors transmit the laser light perpendicular to optical fiber axis. Radial type optical fiber deflectors are ideally suited for laser tissue ablation, because conically shaped optical fiber end transmit the laser energy radially and the laser energy is homogeneously distributed into a ring-shaped beam [8], [9].

The basis of endovenous laser ablation (EVLA) is treatment of venous insufficiency by eliminating the great saphenous

Manuscript received July 28, 2018; revised November 23, 2018; accepted December 20, 2018. Date of publication December 24, 2018; date of current version January 21, 2019. This work was supported in part by TUBITAK under Project 3140846 and Project 7120819. (Corresponding author: B. Ortaç.)

A. A. Seymen is with the E-A Teknoloji LLC, 06800 Ankara, Turkey, also with the Department of Physiology, Erciyes University, 38039 Kayseri, Turkey, and also with the National Nanotechnology Research Center, Institute of Materials Science and Nanotechnology, Bilkent University, 06800 Ankara, Turkey (e-mail: aytac@eateknoloji.com).

E. U. Şimşek and B. Ortaç are with the National Nanotechnology Research Center, Institute of Materials Science and Nanotechnology, Bilkent University, 06800 Ankara, Turkey (e-mail: elifuzcengiz@bilkent.edu.tr; ortac@ unam.bilkent.edu.tr).

E. Özgür is with the E-A Teknoloji LLC, 06800 Ankara, Turkey (e-mail: erol@eateknoloji.com).

Color versions of one or more of the figures in this letter are available online at http://ieeexplore.ieee.org.

Digital Object Identifier 10.1109/LPT.2018.2889594 vein (GSV) using laser energy [10], [11]. There are various reports about using bare [12]-[14] and radial optical fiber deflectors [8], [15]. Even though both fiber deflectors could be used for EVLA; however, the efficiency and the effectiveness of these deflectors is an issue in terms of postoperative pain and bruising [10]. Bare fiber deflectors cause the carbon patch formation on the fiber end [16]. The carbon coated fiber deflector absorbs more of the laser light; therefore, the temperature of deflector increases more than $1200^{\circ} \mathrm{C}$ and it interacts with the vein walls, causing serious temperature increase [17]. On the other hand, ring-shaped beams are more ideal compared to the bare optical fiber deflectors for ablation of cylindrical body parts such as veins, since their homogeneous circular beam leads to equivalently homogeneous ablation of vein walls, and they are prone to cause less temperature rise on the tissue [17]. Different radial emitting light fiber deflector fabrication techniques are proposed in the literature [9], [18], [19].

In addition to these types of deflectors, dual ring or double ring emitting optical fiber deflectors also attract attention for EVLA applications [17]. They enable distribution of the laser energy into two separate ring-shaped circular beams. Having two beams and distributed laser energy, the venous temperature rise is minimized. Moderate temperature changes are observed and high temperature change zone is shrank with different lasers wavelengths [17]. In this concept, the first ring preheats the vein walls, and the second ring ablates the heated surface area more effectively. Although the double ring optical fiber deflector is preferable over single ring to use in EVLA, providing considerable advantages because the laser intensity distribution for each ring decreases the temperature rise inside the vein, resulting in a decreased pre-shrinkage effect, the fabrication process for such fiber deflectors is not well-established. To our knowledge, there is only one patent that proposed a fabrication process for double ring optical fiber deflector containing two conical fiber tips with different cone angles spliced together [20]; however, this process involves advanced polishing and splicing procedures. In this study, we report the first demonstration of double ring firing single optical fiber deflector. A convergent cone geometry having two different cone angles have been developed on a single fiber tip by conventional mechanical polishing technique. Incoming laser light is equally split and separated into two well-defined circular rings due to total internal reflection and refraction at $60^{\circ}$ and $85^{\circ}$ on the structure. Two deflected circular beams with the cone angles of $56^{\circ}$ and $72^{\circ}$ at one single fiber deflector are successfully achieved. The fabrication process of the fiber deflector relies solely on this prominent fiber tip manufacture technique. 


\section{Design and Simulation of Double Ring Firing Single Optical Fiber Deflector}

Circular beam deflection is provided by the conical shaped optical fiber deflectors. The design of double ring firing single optical fiber deflector is presented in Fig. 1. The twoangled $(\theta 1$ and $\theta 2)$ convergent conical fiber deflector geometry is proposed in a single optical fiber tip. The relations between deflection angles $(\phi 1$ and $\phi 2)$ and cone angles $(\theta 1$ and $\theta 2)$ are calculated by using Snell's law and ray tracing approach. There are three light deflection scenarios for the conical shaped optical fiber deflector [9], [21]. In the first scenario (RAY-1), the incoming rays (represented as ray I and ray II) are reflected from the first core-air boundary and then they refracted through outside of the optical fiber for the condition of $\theta_{\text {cone }}<$ $\pi-2 \theta_{\text {core-air. }}$ To provide total internal reflection (TIR) from the first core-air boundary, the critical angle $\theta_{\text {core-air }}$ is then calculated as $\theta_{\text {core-air }}=\sin ^{-1}\left(\mathrm{n}_{\text {air }} / \mathrm{n}_{\text {core }}\right)$ which equals to $43.3^{\circ}$ where the tip angle in the range of $\theta_{\text {cone }}<\pi-2 \theta_{\text {core-air }}$ for obtain side deflection. Then, the reflected ray travels through the second core-air boundary and the angle between surface normal and incoming ray becomes $\omega_{1}$ and it is calculated by $\omega_{1}=\left(2 \theta_{1} / \theta_{2}-180\right) / 2$ from the geometry. The Snell Law is applied at the second boundary and the first deflection angle $\phi_{1}$ is obtained in Eq. 1:

$$
\phi_{1}=\frac{180-\theta_{1}}{2}-\sin ^{-1}\left(\frac{n_{\text {core }}}{n_{\text {air }}} \sin \left(\omega_{1}\right)\right)
$$

In the second scenario (RAY-2), the incoming ray is totally reflected from the first boundary, then it is refracted from the second boundary with the angle $\boldsymbol{\omega}_{\mathbf{2}}=\mathbf{3} \boldsymbol{\theta}_{\mathbf{2}} / \mathbf{2}$. Snell's law is applied at this boundary and the second deflection angle is written as Eq. 2:

$$
\phi_{2}=\frac{180-\theta_{2}}{2}-\sin ^{-1}\left(\frac{n_{\text {core }}}{n_{\text {air }}} \sin \left(90-\omega_{2}\right)\right)
$$

The graphical representation of the relations, between cone angles and deflection angles shown in Fig. 1, can be calculated by using to Equation 1 and Equation 2 where $\mathrm{n}_{\text {core }}$ is 1.45 at the laser wavelength of $635 \mathrm{~nm}$. In our proposed structure, we used the cones angles respecting the condition of $\theta_{\text {cone }}<$ $\pi-2 \theta_{\text {core-air }}$ of $\theta_{1}=56^{\circ}$ and $\theta_{2}=72^{\circ}$, respectively. Our approach clearly demonstrates that two incoming laser lights (ray I and ray II) are refracted through the second coreair boundary with different deflection angles $\left(\phi_{1}\right.$ and $\left.\phi_{2}\right)$ showing two separated circular rings. Other angle pairs such as $\theta 1=52^{\circ}$ and $\theta 2=76^{\circ}$, or $\theta 1=48^{\circ}$ and $\theta 2=82^{\circ}$ also give similar results; however, these values are beyond the working parameters of the polishing machine.

Numerical simulation is also performed to investigate the deflection properties of the proposed structure. Non-sequential design in Zemax software was used for ray tracing analysis of our design. We considered and used commercially available optical fibers (Thorlabs - FT600EMT), both in simulations and experiments. Fiber geometry was constructed with the radius of the core/cladding as $600 / 630 \mu \mathrm{m}$, respectively. The numerical aperture (NA) of the fiber was 0.39 . The rays are coupled inside the fiber core (by using fiber.dll source in Zemax).

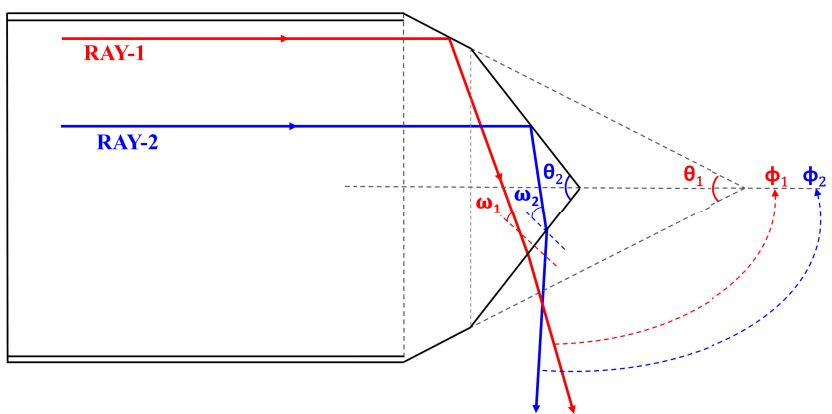

Fig. 1. Design and ray tracing paths for the proposed double ring firing optical fiber deflector.

100,000 rays were used for analysis but only the 100 of the rays are presented in the numerical studies.

The general behavior of simulation configuration is represented in Fig. 2. To cover and study the two circular beam deflection, a $60 \mathrm{~mm}$ diameter semi-spherical detector was used. The fiber was placed in the center of the semi- spherical detector. The incoming rays are refracted from the deflector and they form two circular beam shapes. The two deflected beams are clearly observed at the detector. The first ring deflecting with the angle $\phi_{1}$ equal to $60^{\circ}$ and the second circular beam with the deflection angle $\phi_{2}$ equal to $85^{\circ}$ are shown and this result is in a good agreement with the calculation presented in Equations. In Fig. 2b, the image of the two homogeneous and well separated circular beams is demonstrated at the semi-spherical detector.

\section{EXPERIMENTS AND RESULTS}

Double ring firing single optical fiber deflector fabrication consists of two mechanical polishing steps. In the mechanical polishing process, the deflector geometry (cone shape and angle) is formed with rough lapping film, then, the surface roughness of the deflector is gradually smoothed by polishing with smoother lapping films in order to obtain nanoscale surface roughness [22]. This process is composed of several steps to obtain a high-quality surface structures and a wellprepared fiber deflector surface eliminates the optical losses such as scattering and back reflection. In this study, we used polishing films with four different grit sizes: $5 \mu \mathrm{m}$ grit using silicon carbide, 3 and $1 \mu \mathrm{m}$ grits using aluminum oxide, and $0.3 \mu \mathrm{m}$ grit using calcined alumina (Thorlabs - LF5P, LF3P, LF1P and LF03).

The fabrication approach of the proposed double firing deflector structure is represented in Fig. 3. The first conical shape fiber deflector was formed by one-step mechanical polishing process using mechanical polishing machine (Ultrapol, USA). In Fig. 3a, a bare fiber is located with an angle, $\alpha$ (for the first cone angle, we prefer to use the cone angle of $56^{\circ}$ ) and the fiber rotate around its axis while the polishing film also rotate around its axis in order to obtain conical fiber tip shape. The cone shaped fiber deflector is then smoothened by fine mechanical polishing (see Fig 3b). This type of deflectors provides one circular light beam [9]. In order to develop double circular light deflection, the deflector is polished subsequently with different polishing angle $\beta$ (for the 


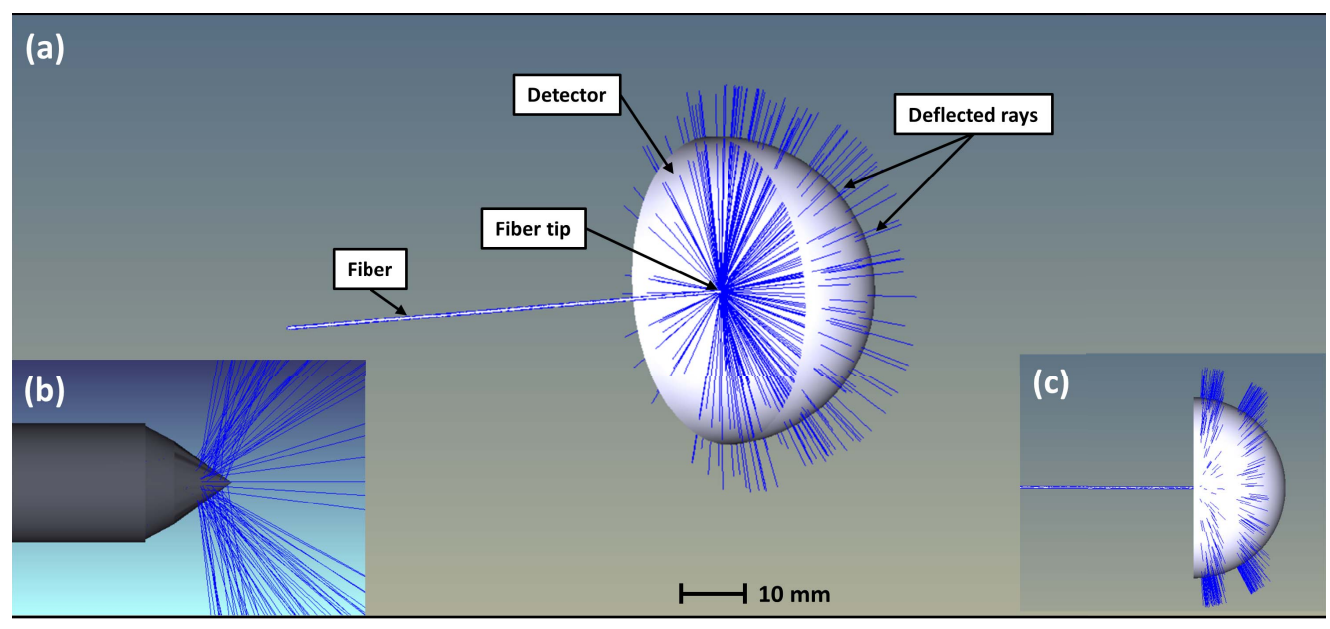

Fig. 2. Numerical simulation results. (a) Light deflection from the double ring firing fiber deflector, (b) ray tracing paths for proposed fiber tip, and (c) light deflection from double ring fiber deflector on semi-spherical detector showing the pattern with two deflection angles.
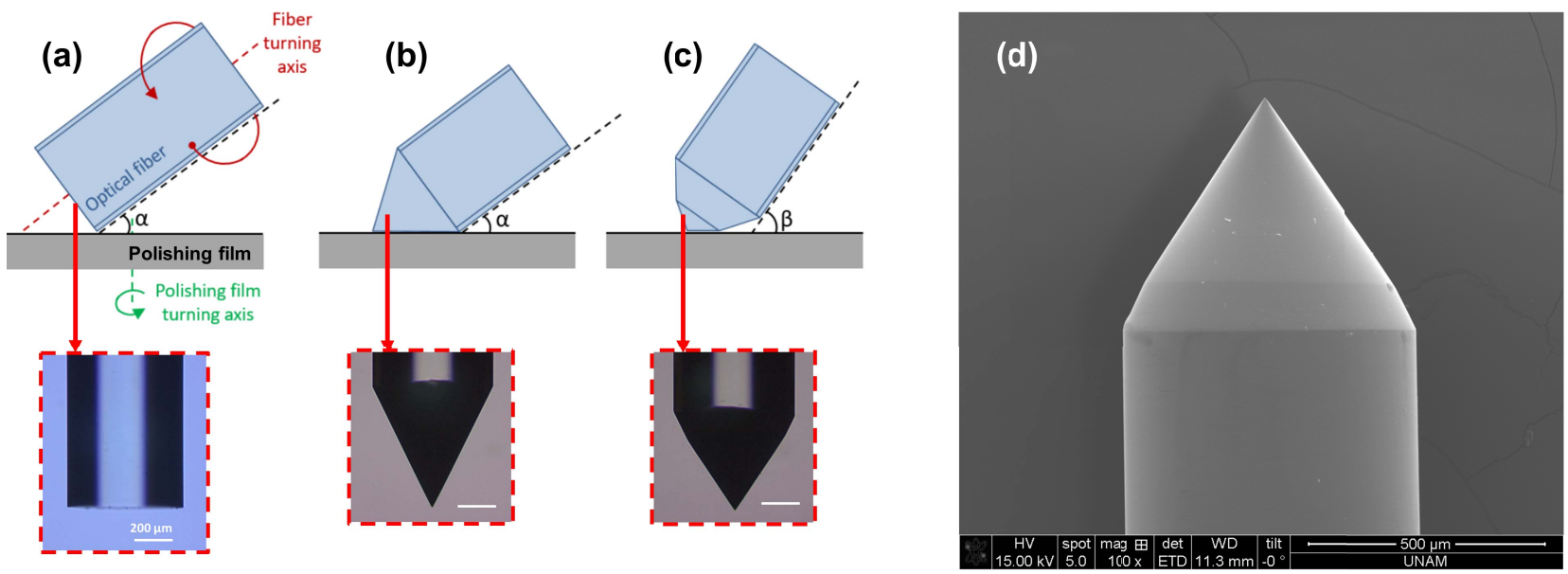

Fig. 3. Fabrication method of double ring firing fiber optical deflector. (a) Polishing technique for fabrication of conical fiber tip, (b) the tip shape obtained after the first polishing step, (c) the tip shape obtained after the second polishing step, and (d) SEM image of the final structure.

second cone angle, we prefer to use the cone angle of $\left.72^{\circ}\right)$ as represented in Fig. 3c. To avoid any optical losses (scattering and back reflection) from second cone shape, fine mechanical polishing is also applied. In this section, we draw special attention to the ratio of the cone heights which effect to the laser energy distribution between two conical areas. In order to obtain equal light distribution, we determine the ratio of cone heights based on our numerical simulation. The best values regarding the equal optical power distribution were obtained when the ratio of the height of the top cone to the bottom cone is approximately 2 , at the previously given angles. The optical microscope images of the final product are shown in Fig. 3c. The surface and shape of the tip was characterized by using a scanning electron microscope (SEM) and as shown in Fig. 3d, two cones shape having different cone angles are distinguished in a single optical fiber tip.

The results of double ring light deflection obtained from final optical fiber deflector product are shown in Fig. 4. The images of laser emission \& deflection at the fabricated deflector are clearly demonstrate two beam deflections from the single optical fiber deflector (Fig. 4a) and well separated circular pattern of the beams on the cylindrical wall of the opaque screen (Fig. 4b). As shown in Fig. 4, we can clearly see that homogeneous laser distribution into 2 ring-shaped beams was formed. In order to analyze the light deflection in detail, the spatial light distribution of the fiber deflector was measured by a home-built characterization set-up represented in Ref. 9 . The normalized intensity vs. deflection angle graph of the fiber deflector was demonstrated in Fig. 4c. The two light deflection angles were measured at $60^{\circ}$ and $85^{\circ}$. The experimental results of the deflection angle positions match well with the calculation and our numerical simulation. We also demonstrated in Fig. 4c that the laser intensity distribution and full width at half maximum for the deflection angles of two conical areas are very close for all direction. Here, we point out that this approach presents several potential advantages. For example, the deflection angles of two cones with different intensity distribution could be adaptable which is important for the real-world applications especially for control the preshrinkage effect in EVLA operations. 

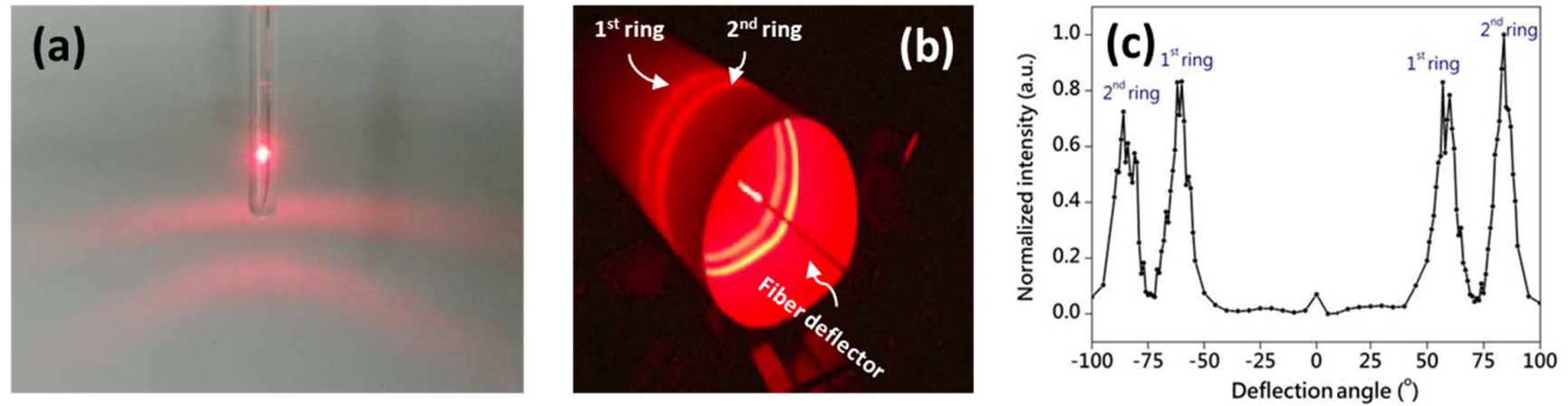

Fig. 4. Demonstration of (a) two beam deflected from the optical fiber, (b) circular pattern of the beams, and (c) spatial optical energy distribution of fiber tip deflecting two circular beams. The laser used in this study was a fiber-coupled Fabry-Perot laser diode source at wavelength of $635 \mathrm{~nm}$ and $2 \mathrm{~mW}$ average output power.

\section{CONCLUSiOnS}

A novel fabrication technique of double ring firing single optical fiber deflector is demonstrated. The design of this deflector is first analyzed by using the ray tracing methods and then the fabrication process is presented in detail. The fabrication process includes only conventional mechanical polishing method. The double ring beam is obtained with two cone angles of $56^{\circ}$ and $72^{\circ}$. This type of optical fiber deflectors is preferred by EVLA operations to overcome problems such as postoperative pain and bruising. We proposed a facile and convenient fabrication method and we achieved two circular beams in one optical fiber tip without complex processes such as cleaving and splicing of polished fiber tips.

\section{ACKNOWLEDGMENT}

We gratefully acknowledge the support of the Vamet Medikal Inc. A patent application concerning the results presented in this report is issued. The authors thank Mine Ertok, Onur Çelik, Arif Doğan, Baran Utku Tekin, Ali Karatutlu and Yakup Midilli for the contributions to this study.

\section{REFERENCES}

[1] S. G. Bown, "Phototherapy in tumors," World J. Surg., vol. 7, no. 6, pp. 700-709, Nov. 1983.

[2] A. Katzir, Lasers and Optical Fibers in Medicine. New York, NY, USA: Academic, 1993.

[3] C. F. P. van Swol, R. M. Verdaasdonk, R. J. van Vliet, D. G. Molenaar, and T. A. Boon, "Side-firing devices for laser prostatectomy," World J. Urol., vol. 13, no. 2, pp. 88-93, Apr. 1995.

[4] G. J. Tearney et al., "In vivo endoscopic optical biopsy with optical coherence tomography," Science, vol. 276, no. 5321, pp. 2037-2039, Jun. 1997

[5] Z. Wang and N. Chocat, "Fiber-optic technologies in laser-based therapeutics: Threads for a cure," Current Pharmaceutical Biotechnol., vol. 11 , no. 4, pp. 384-397, Jun. 2010.

[6] O. Göckeritz, "Current standards and recent progress in minimally invasive phlebo surgery," J. Cutaneous Aesthetic Surg., vol. 5, no. 2, pp. 104-114, Apr./Jun. 2012.

[7] G. Keiser, F. Xiong, Y. Cui, and P. P. Shum, "Review of diverse optical fibers used in biomedical research and clinical practice," Proc. SPIE. vol. 19, no. 8, p. 080902, Aug. 2014.
[8] F. Pannier, E. Rabe, J. Rits, A. Kadiss, and U. Maurins, "Endovenous laser ablation of great saphenous veins using a $1470 \mathrm{~nm}$ diode laser and the radial fibre-Follow-up after six months," Phlebology, vol. 26, no. 26, pp. 35-39, Feb. 2011.

[9] E. U. Şimşek, B. Şimşek, and B. Ortaç, " $\mathrm{CO}_{2}$ laser polishing of conical shaped optical fiber deflectors," Appl. Phys. B, Lasers Opt., vol. 123, no. 6, p. 176, May 2017

[10] L. Mundy, T. L. Merlin, R. A. Fitridge, and J. E. Hiller, "Systematic review of endovenous laser treatment for varicose veins," Brit. J. Surg., vol. 92, no. 10, pp. 1189-1194, Sep. 2005.

[11] R. R. van den Bos, M. Neumann, K.-P. De Roos, and T. Nijsten, "Endovenous laser ablation-induced complications: Review of the literature and new cases," Dermatol. Surg., vol. 35, no. 8, pp. 1206-1214, Aug. 2009.

[12] T. M. Proebstle et al., "Endovenous treatment of the greater saphenous vein with a 940-nm diode laser: Thrombotic occlusion after endoluminal thermal damage by laser-generated steam bubbles," J. Vascular Surg. vol. 35, no. 4, pp. 729-736, Apr. 2002.

[13] R. J. Min, N. Khilnani, and S. E. Zimmet, "Endovenous laser treatment of saphenous vein reflux: Long-term results," J. Vascular Intervent. Radiol., vol. 14, no. 8, pp. 991-996, Aug. 2003.

[14] L. S. Kabnick and J. A. Caruso, "No-wall touch laser fiber vs baretip laser fiber for endothermal venous ablation of great saphenous vein: Are the results the same?" in Controversies Updates Vascular Surgery, J. L. Gerard, Ed. Torino, ON, Canada: Edizioni Panminerva Medica, 2008, pp. 401-402.

[15] K. Stock, T. Stegmayer, R. Graser, W. Förster, and R. Hibst, "Comparison of different focusing fiber tips for improved oral diode laser surgery," Lasers Surg. Med., vol. 44, no. 10, pp. 815-823, Dec. 2012.

[16] M. M. Amzayyb et al., "Carbonized blood deposited on fibres during 810, 940 and 1,470 $\mathrm{nm}$ endovenous laser ablation: Thickness and absorption by optical coherence tomography," Lasers Med. Sci., vol. 25, no. 3, pp. 439-447, May 2010.

[17] T. Yamamoto and M. Sakata, "Influence of fibers and wavelengths on the mechanism of action of endovenous laser ablation," J. Vascular Surg. Venous Lymphatic Disorders, vol. 2, no. 1, pp. 61-69, Jan. 2014.

[18] I.-B. Sohn, Y. Kim, Y.-C. Noh, I. W. Lee, J. K. Kim, and H. Lee, "Femtosecond laser and arc discharge induced microstructuring on optical fiber tip for the multidirectional firing," Opt. Express, vol. 18, no. 19, pp. 19755-19760, Sep. 2010.

[19] S. H. Lee et al., "Radial-firing optical fiber tip containing conical-shaped air-pocket for biomedical applications," Opt. Express, vol. 23, no. 16, pp. 21254-21263, Aug. 2015.

[20] W. Neuberger and A. Harschack, "Endoluminal laser ablation device and improved method for treating veins," U.S. Patent 036095, May 11, 2011.

[21] T. Watanabe and Y. Matsuura, "Side-firing sealing caps for hollow optical fibers," Lasers Surg. Med., vol. 38, no. 8, pp. 792-797, Aug. 2006.

[22] J. M. Bennett and R. J. King, "Effect of polishing technique on the roughness and residual surface film on fused quartz optical flats," Appl. Opt., vol. 9, no. 1, pp. 236-238, Jan. 1970. 